\title{
Les SHS à la croisée des chemins
}

Introduction

Human and social sciences at the crossroads

Michel Lussault

\section{CpenEdition} Journals

Édition électronique

URL : http://journals.openedition.org/ries/554

DOI : $10.4000 /$ ries.554

ISSN : 2261-4265

Éditeur

Centre international d'études pédagogiques

Édition imprimée

Date de publication : 1 décembre 2008

Pagination : 21-30

ISSN : $1254-4590$

Référence électronique

Michel Lussault, «Les SHS à la croisée des chemins », Revue internationale d'éducation de Sèvres [En ligne], 49 | décembre 2008, mis en ligne le 28 juin 2011, consulté le 03 mai 2019. URL : http:// journals.openedition.org/ries/554; DOI : 10.4000/ries.554

(c) Tous droits réservés 


\title{
Quel avenir pour les études en sciences humaines?
}

\section{Introduction}

\section{Les SHS à la croisée des chemins}

\author{
Michel Lussault
}

\section{UN ÂPRE DÉBAT}

Les questions liées de l'enseignement des sciences humaines et sociales (SHS) et de l'avenir de celles-ci sont régulièrement le sujet de débats, souvent passionnés et contradictoires. Schématiquement, on peut estimer que ces débats, quels que soient les pays où ils adviennent, recouvrent trois types d'interrogations - au vrai complémentaires et peu dissociables et qui ne sont ici séparées que par l'exigence de clarté de la présentation :

- Qu'en est-il précisément de ce domaine de la connaissance nommé sciences humaines et sociales par les uns, humanités par d'autres (ce dernier mot revenant ces temps-ci au goût du jour), ou encore lettres, etc. ? Ainsi, le choix d'un intitulé pour circonscrire un tel champ de connaissance n'est pas trivial et cette dispute montre d'ailleurs que l'organisation de ce champ est encore un enjeu. En particulier, lorsqu'on privilégie l'approche en termes de sciences sociales, on participe d'un autre rapport aux objets de connaissance, aux méthodes d'investigation, aux formes de structuration de la recherche que lorsqu'on se place dans la perspective des "humanités ». Un tel débat possède une dimension internationale, car les sémantiques ne sont pas les mêmes dans les différentes langues et les traditions académiques sont aussi fort variées.

- Quelle que soit la nomenclature utilisée, bien des discussions se cristallisent sur le problème des finalités des disciplines ici évoquées. S'agit-il de purs savoirs d'érudition, attachés au seul ciel des idées? Doit-on y voir des connaissances critiques à fortes implications sociales et politiques, ce qui exigerait qu'on écarte toute préoccupation instrumentale de professionnalisation, de "soumission" à une logique externe, quelle qu'elle soit? Ou faut-il a contrario insister sur l'employabilité d'un individu, dès lors qu'il maîtrise les savoirs et compétences des «SHS» ou humanités?

- À l'heure où l'évolution de l'enseignement public est, dans de nombreux pays, l'objet de bien des interrogations, comment doit-on aujourd'hui envisager les apprentissages de ces «matières» tant dans le secondaire que 
le supérieur? Quels contenus privilégier, quelles méthodes choisir, comment évaluer les étudiants, etc. ?

Dans un certain nombre de pays où les filières de SHS furent ou sont encore touchées par une massification notable et une augmentation rapide du nombre d'étudiants (comme en Italie, en Espagne, en France, au Maghreb, en Afrique), ce débat prend souvent un tour catastrophiste. On évoque une crise, on dépeint un avenir sombre (voire pas d'avenir du tout!) pour tout ce que l'on rassemble sous la bannière des filières de sciences humaines et sociales. Cellesci, pas toujours dotées, loin de là, de moyens financiers à la hauteur des besoins, accueillant de nombreux jeunes issus de milieux sociaux peu favorisés, apparaissent fréquemment comme de moins en moins aptes à offrir une véritable perspective pour les étudiants et on met en question leur pertinence pour former des individus à trouver leurs voies. Il va de soi qu'une telle approche ne se constate sans doute pas partout (à Harvard, les humanités sont prisées et leurs débouchés prestigieux!) et c'est d'ailleurs un enjeu de connaissance important que d'apprécier cette différenciation.

Constatant à la fois l'ampleur et la généralité de ces interrogations, la présence des SHS dans la plupart des situations académiques et la variété de leurs statuts et de leur réputation, la Revue internationale d'éducation de Sèvres a décidé de consacrer un numéro à cette question - au demeurant très rarement abordée de manière systématique. C'est en effet un des premiers constats que nous avons réalisés, et qui nous a surpris, tant nous pensions qu'une telle thématique était déjà bien balisée. Or il n'en est rien et cela s'est traduit par quelques difficultés.

Tout d'abord, il s'est avéré relativement malaisé, et pas seulement en France, de trouver des spécialistes des questions d'enseignement des sciences humaines et sociales, c'est-à-dire des hommes et des femmes qui travaillent «à froid» sur ces champs, en proposant un panorama appuyé sur de véritables matériaux empiriques, sur une vraie problématique, sur une épistémologie claire. S'il existe de nombreux chercheurs qui travaillent sur les politiques publiques éducatives, ils n'abordent en général les SHS qu'incidemment, à travers le prisme, par exemple, de l'analyse de l'explosion démographique des effectifs de l'enseignement supérieur dans certaines filières des SHS. D'autres ne traitent que des questions d'épistémologie, d'histoire, d'anthropologie des savoirs des sciences humaines, sans se consacrer aux problématiques de l'organisation institutionnelle et sociale de leur transmission, d'autres encore se focalisent sur les analyses didactiques, en laissant de côté, eux aussi, les points que ce numéro souhaitait aborder. Bref, si l'écho social de l'interrogation sur l'avenir de l'enseignement des SHS est bien sonore, il ne s'agit pas pour autant d'une priorité scientifique. C'est un sujet dont on parle plus, dans le registre de l'opinion, qu'on ne l'étudie vraiment. 
Ensuite, et sans doute conséquemment, nous n'avons pas trouvé d'auteurs volontaires pour traiter de la situation de grands pays d'enseignement supérieur, comme la Grande-Bretagne, l'Allemagne, les États-Unis : les nombreux collègues contactés, tous éminents dans leurs domaines, ne s'estimaient en général pas capables, car non spécialistes, "véritablement», de la question, de proposer une analyse générale de la situation desdits pays dans le cadre contraint d'un article. Ceci nous a confirmé que notre numéro ne couvrait pas une problématique bien cernée et reconnue dans le champ académique. Enfin, et à notre grand regret, nous n'avons pas suscité de nombreuses vocations en ce qui concerne l'examen des sciences humaines et sociales dans l'enseignement secondaire, ce qui aurait permis de traiter plus intensément de la relation entre celui-ci et l'enseignement supérieur.

Tout ceci nous a amenés à resserrer notre dossier autour d'études de cas originales, conçues comme une invitation au lecteur à entrer dans une série de problématiques particulièrement complexes. Ce qui frappe, à lire les articles, c'est qu'ils nous confrontent toutes à des questions qui renvoient aux fondements même de la réflexion sur la finalité de l'enseignement supérieur, comme si parler des SHS dans une perspective non exclusivement épistémologique consistait toujours à s'en échapper pour aborder des phénomènes sociaux et politiques globaux majeurs. En particulier, si l'appel à contribution lancé pour constituer ce dossier poussait les auteurs à se demander quel était l'avenir des humanités, on s'est aperçu que tout nous ramenait en fait à une série d'interrogations, encore ouvertes et non tranchées, loin s'en faut, sur leur rôle actuel dans l'économie des savoirs (fondamentaux et appliqués) et dans la dynamique sociale et politique. Comment, dès lors, parler d'avenir alors que tout paraît encore très embrouillé, fragmentaire, contradictoire? Sauf à envisager la disparition des SHS comme seul avenir possible, qu'on s'en attriste ou s'en réjouisse, ou à ne jamais se poser la moindre question, deux attitudes au demeurant très répandues.

\section{UN CHAMP DE FORCES}

À bien y réfléchir, on peut schématiquement discerner quatre positions «idéal-typiques» par rapport aux sciences humaines et sociales et à leurs finalités, qui pèsent fortement sur les débats autour de l'enseignement et de la recherche dans ces disciplines. Chacune de ces postures identifie un placement possible dans un champ de force.

1. La première, patrimoniale et érudite, est celle qui consiste à considérer principalement, voire exclusivement, les SHS sous leur aspect de savoirs d'érudition et de connaissances académiques pures, constituant intrinsèquement un patrimoine à sauvegarder et archiver. Il s'agit là d'affirmer que ces disciplines sont et doivent rester un sanctuaire, hors des turbulences des temps. Et ce, à la fois parce que la connaissance est un absolu, et parce qu'elles fondent 
la culture de «l'honnête homme» occidental. Et il est vrai que les humanités, plus encore que les sciences sociales, constituent un des creusets où se forgea l'identité du sujet cultivé classique. Ce n'est pas un hasard si les cultures scolaires d'élite de bien des pays européens et des États-Unis ont longtemps privilégié les lettres et les humanités. Il reste aujourd'hui encore bien des traces de cette prégnance, même si d'autres formes de distinction sociale que celle permise par la culture classique et d'autres modes d'affirmation du capital scolaire s'imposent. Une telle posture assigne aux filières de SHS une double mission essentielle de conservation et de transmission. Celle-ci consiste à former des enseignants et des chercheurs qui pourront reprendre un tel flambeau, assumer l'antique filiation. Cette stratégie de reproduction d'héritiers confronte de facto les universitaires qui y adhèrent à un malaise grandissant devant la croissance des effectifs étudiants, qui place bon nombre de ceux-ci dans une impasse et semble briser le pacte implicite multiséculaire entre les maîtres et leurs élèves que beaucoup de clercs imaginent perpétuer.

2. La seconde attitude n'est pas réductible à la première, même si elle la recoupe. Elle postule que les SHS constituent le socle de connaissances qui permet d'intégrer et de comprendre (au sens étymologique du mot) tous les autres savoirs et phénomènes du monde. Cette posture, qui réactualise le débat, né au XIX ${ }^{\mathrm{e}}$ siècle, au moment de la constitution de l'Université moderne appelé "conflit des facultés", et qu'examine Alain Renaut dans sa contribution à ce numéro, me semble aujourd'hui comporter deux variantes. La première, que je nommerai variante critique, donne aux SHS un rôle de conscience critique de la société contemporaine. Ces savoirs, inventés et institués en Europe au $\mathrm{XIX}^{\mathrm{e}}$ siècle en tant que disciplines universitaires et scientifiques, et qui de ce fait même ont contribué à définir le profil de l'individu occidental contemporain, comme les humanités ont forgé l'homme classique, serviraient à dévoiler les apparences, à traquer les mirages, à dénoncer les tromperies que les sociétés mettent en avant pour masquer les processus d'aliénation et d'exploitation qu'elles entretiennent. Une telle posture de surplomb est fréquente, et pousse à postuler que les SHS ont une place à part dans la société. Cette attitude repose sur une vision très particulière de l'organisation sociale, qui serait par essence aliénante, et incite à développer un discours «radical», qui se manifeste par une fréquente dénonciation des volontés des pouvoirs en place de museler, voire de faire disparaître des savoirs dangereux pour l'ordre établi. La seconde variante que je baptise réflexive, insiste plutôt sur le rôle des SHS dans l'acquisition par les acteurs sociaux et leurs collectifs d'une réflexivité indispensable à la compréhension tant des ressorts de l'action individuelle que des logiques de la dynamique sociale. Cette tendance est moins agonistique que la précédente, plus empirique et scientifique; elle peut même, dans de nombreux domaines, fonder des savoirs d'expertise proposés aux intervenants sociaux.

3. La troisième posture est prosaïque. Elle affecte aux SHS un rôle de cadre de culture générale. Il ne s'agit là ni de se recueillir devant le tabernacle 
de la culture érudite de l'élite, ni de communier dans la ferveur des grandes messes critiques, ni de réfléchir l'individu postmoderne, mais de reconnaître que ces savoirs pourvoient des compétences génériques nécessaires à toute personne souhaitant s'insérer socialement et économiquement. Cette attitude, très courante, dans l'enseignement secondaire, comme dans certains systèmes d'enseignement supérieur, tend à banaliser les savoirs des sciences humaines et sociales, alors que les deux précédentes, chacune différemment, les héroïsaient.

4. La quatrième posture est plus explicitement utilitariste que la précédente. Elle confère aux SHS une fonction d'instrument, plus ou moins important, au service d'autres finalités principales. Là encore, je distingue deux variantes. L'une fait des humanités le pourvoyeur d'un vernis culturel, d'un supplément d'âme de formations autres, comme celles d'ingénieurs. On tend à nommer ce vernis, souvent sans trop y penser : culture générale - oubliant au passage que la notion de culture générale est tout sauf une évidence anhistorique. Ici, les humanités servent d'auxiliaire, comme par exemple ces modules de sciences humaines dans bien des filières médicales, censés apporter une coloration d'éthique au cursus. L'autre prend le parti de considérer que les SHS doivent être les supports d'une ingénierie sociale et économique spécifique et traitent les savoirs dans cette perspective d'insertion professionnelle des étudiants dans des champs de métiers spécialisés. On trouve cette propension dans les disciplines juridiques, économiques, de gestion, mais aussi en matière d'aménagement, de langues, de tourisme, voire de valorisation de patrimoine historique. Une telle pente utilitariste est décelable dans les pays de vieille tradition universitaire, où pourtant les savoirs érudits des humanités, constitutifs de l'épistémè contemporaine et des cultures scolaires et sociales de références, restent présents et où les sciences sociales continuent d'avoir une réelle légitimité réflexive et critique. Là, bien des voix (celles des gouvernements, des entreprises, des familles, des étudiants) se font entendre pour que les SHS prennent le tournant de la professionnalisation. Des filières nouvelles se développent pour ce faire. Mais fréquemment, ces évolutions fixent la désapprobation des tenants encore nombreux (enseignants, étudiants) des deux premières positions, qui refusent ce qu'ils considèrent comme une dérive préjudiciable à la dynamique des savoirs "désintéressés", comme une tyrannie de la logique de marché qui menace la liberté de penser et d'étudier. Dans des contrées comme la France ou l'Italie, il n'est pas rare qu'un tel conflit des opinions débouche sur des crises et des grèves paralysant le système universitaire. C'est dans les pays en croissance forte et dans les pays émergents des continents asiatique, africain et américains qu'on trouve la présence la plus marquée de la conception instrumentale. De très nombreux étudiants y choisissent des parcours de droit, de management, d'économie, de business, de relations internationales, de langues étrangères appliquées, pour leurs capacités, réelles ou supposées, à apporter une formation exploitable rapidement sur le marché national ou/et international du travail. Il n'est pas rare que cette évolution soit accentuée par 
la méfiance des pouvoirs politiques vis à vis de disciplines de SHS aux registres plus critiques, pouvoirs politiques qui n'hésitent pas à contrôler des appareils universitaires alors soumis à la double pression d'une férule institutionnelle et d'une dynamique concurrentielle du marché de la formation qui pousse à anticiper les désirs de l'étudiant-consommateur.

Il faut noter que les approches érudites et critiques ont plutôt tendance à valoriser la structuration de l'enseignement supérieur, dès après la sortie de l'enseignement secondaire, en filières disciplinaires strictement séparées et à repousser l'idée de proposer des enseignements plus transversaux et mélangés au motif que ceux-ci affaibliraient la science au profit d'une conception utilitaire. Alors que là où les sciences humaines et sociales participent plutôt des positions trois et quatre, les parcours de formation sont beaucoup moins rapidement spécialisés, plus généralistes, comme le montre le cas extrême des universités des États-Unis, où la spécialisation, si elle arrive, est très tardive. Du coup, il n'est pas rare que les expérimentations pédagogiques et didactiques soient plus notables dans les filières explicitement professionnalisantes ou de culture générale que dans celles qui revendiquent la qualité et l'autonomie critique de la science. Il y a là, à mes yeux, un paradoxe qui ne compte pas peu dans la désaffection d'une partie du public étudiant pour les parcours les plus académiques, où la nécessité proclamée de l'autonomie de l'élève correspond souvent de fait à un abandon de celui-ci à son sort.

Il n'est bien sûr pas rare qu'un même individu, une même institution, emprunte à chacune de ces quatre conceptions. Par exemple, la GrandeBretagne possède un système, de ce point de vue, très hybride, beaucoup plus que celui de l'Allemagne, encore très marqué par le modèle humboldtien. Ce qui m'importe ici, c'est de souligner que ces approches sous-tendent bien des argumentaires et des positions. Les différents articles de ce dossier ne dérogent pas à cette règle et le lecteur pourra s'amuser à les placer au sein de ce carré des valeurs. Car il s'agit de positions qui manifestent une prise de parti idéologique (au sens non péjoratif du mot) par rapport à la question simple, que pose ce numéro : quel rôle pour les SHS ? Or tenter de répondre à une telle interrogation, c'est immédiatement, compte tenu de ce que sont les sciences humaines et sociales et de leur contribution à la constitution des cadres de références intellectuels des sociétés contemporaines, se projeter à un autre niveau de problèmes, celui de la finalité du système d'enseignement et même de la raison d'être de tout savoir. Et là, l'alternative paraît claire : faut-il considérer que le savoir (et donc sa transmission) possède une finalité intrinsèque, qui n'est pas dépendante d'une autre injonction que celle de la libido sciendi (attitudes un et deux)? Ou doit-on reconnaître que le savoir ne vaut que pour les services qu'il permet de rendre à la collectivité (attitudes trois et quatre)? Il n'est guère aisé de sortir d'un tel duel, qu'on considère assez spontanément comme caricatural, mais dont pourtant on reprend souvent les termes, faute de mieux. 
Heureusement, et de plus en plus, des tentatives de décalage des points de vue et de dépassement des antagonismes se font jour. Ce n'est pas un hasard si nous ouvrons ce dossier avec le texte d'Alain Renaut et le fermons avec celui de Renato Janine Ribeiro. Le premier, à partir d'un rappel de l'épaisseur historique du débat, pose des principes qui ouvrent de nouvelles perspectives pour envisager une réforme des enseignements supérieurs de sciences humaines et sociales autour de la redéfinition de la notion de culture générale. Alain Renaut vise à échapper tant au piège de l'érudition pure qu'à celui de la posture tribunicienne de conscience critique, ou qu'à celui de l'utilitarisme. Il suggère une démarche qui allie exigence intellectuelle, souci de qualité scientifique, approche des compétences à transmettre, éthique de la transmission. C'est ce pari qu'a tenté, à sa manière, Renato Janine Ribeiro en concevant pour l'université de Saõ Paulo un passionnant cursus expérimental en sciences humaines. Le fait que ce projet n'ait pas abouti ne retire rien à sa qualité et à sa portée pour tous ceux que l'enseignement des sciences humaines intéresse.

\section{UN PARCOURS DE RÉFLEXION}

Entre ces deux balises, nous avons disposé les contributions de manière à donner des exemples aussi larges que possibles de types de situation qu'on peut rencontrer. Nulle prétention à l'exhaustivité n'a guidé la constitution de ce numéro et nous avons choisi de nous focaliser sur des situations nationales trop rarement étudiées. Ce faisant, nous n'avons pas manqué notre cible, bien au contraire. Car tous les textes nous confirment la pertinence de la problématique d'ensemble, d'échelle mondiale, concernant la fonction des SHS. Par ailleurs, les cas analysés nous permettent aussi de mesurer l'impact des modèles que constituent la Grande Bretagne et les États-Unis. Ce modèle est à la fois organisationnel, épistémologique, et linguistique. Organisationnel, car ces deux pays proposent des exemples très aboutis de modularisation des cursus, notamment de premier cycle universitaire, lors desquels il n'existe pas réellement de focalisation disciplinaire précoce. Les SHS constituent donc des propositions parmi d'autres, qui sont offertes en tant qu'elles fournissent les bases d'un bagage intellectuel élémentaire, ce qui n'exclut pas de trouver ensuite les spécialisations scientifiques les plus abouties. Épistémologique, car les sciences humaines et sociales anglophones sont aujourd'hui des réservoirs de théories et de concepts particulièrement riches et auxquels de nombreux emprunts sont faits, partout, dans les autres univers académiques. Linguistique, car la langue anglaise devient, pour les SHS aussi, le véhicule de plus en plus prégnant de la diffusion des savoirs scientifiques, que cela soit via les publications ou via les formations, bien au-delà du seul monde anglophone. Enfin, les articles nous montrent tous que, nonobstant la parenté des grands problèmes rencontrés (comme ceux de la croissance des effectifs, de la réflexion sur les finalités des cursus, du financement des parcours de sciences humaines et sociales), chaque situation nationale 
se caractérise par la spécificité des déclinaisons des solutions élaborées par les pouvoirs publics, les universités, les étudiants pour assurer le développement des SHS.

Précisons enfin que nous n'avons pas imposé aux auteurs une définition fixe du champ des SHS, car nous avons pensé que cela risquait de provoquer des réticences et de fixer la réflexion sur un point certes important mais qui ne constituait pas notre cible principale. Les articles balisent eux-mêmes le périmètre qui les concerne, et c'est une façon de signaler que cette question reste ouverte.

Après le texte d'Alain Renaut, qui donne une vision globale de l'évolution des termes du débat à l'échelle de l'Europe, nous avons placé l'analyse de Jesper Eckhardt Larsen qui, prenant l'exemple du Danemark, montre bien la tension entre les partisans de sciences humaines protégées par les remparts académiques et les tenants, très actifs au sein même du gouvernement danois et du monde de l'entreprise, d'une évolution utilitariste. Il nous livre au passage d'intéressants éléments de comparaison avec la Suède et la Norvège et aborde la disjonction entre le statut des sciences humaines dans l'enseignement secondaire et celui dans l'enseignement supérieur. Anita Gramigna, quant à elle, se focalise plutôt sur l'enseignement secondaire en Italie, soumis ces dernières années à de nombreuses réformes destinées à le rendre plus efficace. Elle décrit les fondements du lycée classique, au sein duquel la philosophie joue un rôle essentiel. Elle montre comment la philosophie est aujourd'hui mise en question par la montée en puissance d'autres voies d'enseignement secondaire, appuyées sur des méthodes pédagogiques qui donnent à l'expérience des élèves un rôle crucial. Des sciences humaines plus empiriques peuvent alors jouer leur partition. Cet article ouvre en ce domaine d'intéressantes perspectives qui appellent un prolongement de la réflexion sur les formes possibles de l'activité pédagogique en sciences humaines et sociales. Liudmila Pipiya examine ensuite les mutations récentes de l'enseignement supérieur et de la recherche en Russie. Elle décrit les relations qui existent entre les bouleversements politiques et sociaux russes et les restructurations des SHS dans un pays où, au temps de l'Union soviétique, la recherche était très encadrée politiquement et assurée par des instituts et des académies, organismes extérieurs aux universités. Cette séparation, en Russie comme ailleurs, en France par exemple, ou en Chine, a eu des conséquences lourdes tant sur la vie scientifique que sur la constitution des filières spécialisées de l'enseignement supérieur, et a eu plutôt tendance à affaiblir et désorganiser celui-ci. Cet article, qui est consacré à un cas national très spectaculaire, nous indique donc qu'il est essentiel, lorsqu'on réfléchit aux formations en SHS, de s'intéresser à la fois aux contextes politiques sociaux généraux et aux modes de structuration de l'activité scientifique. Si celle-ci s’inscrit préférentiellement dans les universités, dans des conditions de réelle liberté académique et de bonne dotation financière, l'impact sur la dynamique et l'attractivité de l'ensemble du système d'enseignement et de recherche apparaît positif. 
La table ronde que nous avons consacrée au cas français, dont la transcription est placée au milieu du dossier, est révélatrice de toutes ces tensions et de ces liens complexes entre les formations supérieures, les activités scientifiques, les cadres sociaux, culturels et économiques généraux. On le sait, le «modèle français» est plutôt un contre-modèle, au sens où il s'avère une exception à l'échelle internationale. En la matière, la tension entre universités et écoles d'une part et universités et établissement nationaux de recherche d'autre part est ici portée à son comble. Il n'est pas interdit de la trouver, globalement, autant sclérosante qu'énergisante, mais le but de la table ronde n'était pas de trancher ce débat franco-français. Il s'agissait pour nous, en faisant appel aux réflexions de professionnels de haut niveau, parfaits connaisseurs du système national, d'entendre des témoignages argumentés et appuyés sur une expérience de terrain, permettant de comprendre les forces et faiblesses de l'enseignement des SHS et de tracer des perspectives d'action. Nous avons réuni, à l'invitation de Tristan Lecoq, directeur du CIEP et qui nous a fait l'honneur d'ouvrir la table ronde, France Bessis-Favard, proviseure du Lycée Molière à Paris, le recteur Alain Boissinot, Olivier Faron, directeur de l'École normale supérieure lettres et sciences humaines, Daniel Filâtre, président de l'Université de Toulouse 2 le Mirail, Pierre Tapie, directeur général de l'ESSEC. Le texte restitue bien la qualité et la densité du débat, qui fut vivifié par la liberté de parole de chacun. Il constitue une importante contribution à la réflexion et dont les apports excèdent de beaucoup le seul cas français.

Notre dossier se poursuit par l'article de Yassine Ferfera et Tchirine Mekideche, consacré au cas algérien. Le texte nous donne une analyse de longue durée qui retrace l'ensemble de la période de constitution de l'université postcoloniale en Algérie. Il examine avec beaucoup de précisions la place prise par des filières de SHS confrontées à une croissance vertigineuse des effectifs, ellemême procédant de l'augmentation rapide du nombre de lauréats du diplôme de fin d'études secondaires. La situation algérienne est emblématique de celle qu'ont connue tous les systèmes universitaires relativement récents et en fort développement, qui ont dû, en quelques années, passer de l'accueil d'une toute petite élite sociale à celui des grands nombres, dans le cadre d'un processus de démocratisation de l'enseignement supérieur justifié au demeurant par des arguments variés selon les situations nationales. Ce processus a presque toujours consisté, dans le monde entier, en une massification trop peu maîtrisée, faute de moyens humains et financiers et peut-être de réflexion en amont sur ce que l'enseignement universitaire pouvait et devait apporter. Les filières de SHS furent, en Algérie comme ailleurs, les premières concernées, car on a longtemps voulu croire qu'elles étaient susceptibles d'absorber rapidement des cohortes sans cesse plus importantes sans investissement trop lourd, comparativement aux cursus de sciences expérimentales, de santé et d'ingénierie. Ceux-ci apparaissaient aussi plus difficiles à suivre que les SHS, que bien des décideurs considèrent spontanément comme plus aisées à comprendre, moins 
sélectives, mieux adaptées à la masse. La double erreur de l'affirmation de la facilité supposée du cursus (opinion qui exprime surtout l'ignorance de ceux qui la professent) et $\mathrm{du}$ faible investissement relatif nécessaire se paie aujourd'hui très cher dans tous les pays concernés. L'Algérie fait partie de ceuxlà, mais aussi la France, l'Italie, l'Espagne et bien d'autres.

Le cas kenyan participe aussi de cette évolution, avec des nuances que traitent avec efficacité les deux auteurs, Hélène Charton et Samuel Owuor. Ils nous décrivent une université kenyane soumise elle aussi à la pression démographique, mais dans un contexte où le pouvoir en place joue la carte du développement des filières privées, censé compenser les capacités trop faibles d'un système public sous-dimensionné. L'article constitue une étude précieuse d'un exemple dont la portée dépasse celui du Kenya et emblématise la situation de l'ensemble de l'Afrique de l'Est.

Le dossier, comme il a été déjà signalé, traite ultimement d'un exemple brésilien. La présentation détaillée par Renato Janine Ribeiro du cursus expérimental d'humanités permet de ne pas délaisser un des points qui avait retenu l'attention du comité de rédaction de la revue lors de la réflexion préalable au choix de la thématique du numéro 49 : quelles sont les initiatives prises pour renouveler les routines pédagogiques, dépasser les certitudes didactiques, rompre avec les habitudes disciplinaires? De surcroît, l'article de notre collègue brésilien donne de précieuses indications sur l'évolution de l'université brésilienne.

Comme tout dossier de la Revue internationale d'éducation de Sèvres, celui-ci s'accompagne d'une bibliographie établie par Bernadette Plumelle, précieux outil de travail pour tous ceux qui voudront prolonger la réflexion que nous avons souhaité engager. Bien sûr, notre initiative réclamerait de nombreux compléments : bien des cas passionnants n’ont pas été traités; la comparaison d'ensemble entre toutes les situations nationales devrait être faite; le lien entre l'organisation de la recherche et celle de l'enseignement serait à examiner plus en détail; la variété des cursus de sciences humaines et sociales imposerait une analyse plus systématique et une étude des contenus et objectifs des curricula, ainsi que des pratiques pédagogiques et didactiques; la question de l'insertion des diplômés de SHS resterait à saisir en profondeur. C'est le propre de tout dossier que d'être partiel et insatisfaisant. Mais nous pensons, rétrospectivement, qu'il permet de bien identifier un terrain de recherche particulièrement fécond, dont les implications intellectuelles, culturelles, sociales, économiques et politiques imposent que les scientifiques, les experts et les décideurs s'en emparent pleinement, enfin. 